\title{
Naturopathy Therapy
}

National Cancer Institute

\section{Source}

National Cancer Institute. Naturopathy Therapy. NCI Thesaurus. Code C15283.

A system of therapy based on preventative care, and on the use of heat, water, light, air, and massage as primary therapies for disease. Some naturopaths use no medications, either pharmaceutical or herbal. Some recommend herbal remedies only. A few who are licensed to prescribe may recommend pharmaceuticals in those cases where they feel their use is warranted. (MedicineNet.com) 